Pacific Journal of Mathematics

A WITT'S THEOREM FOR UNIMODULAR LATTICES 


\title{
A WITT'S THEOREM FOR UNIMODULAR LATTICES
}

\author{
Y. C. LEE
}

Let $K$ be a dyadic local field, $o$ its ring of integers, $L$ a regular unimodular lattice over ${ }^{\circ}$. If $x$ and $y$ are vectors in $L$, we ask for necessary and sufficient conditions to map $x$ isometrically to $y$. Trojan and James obtain conditions via a $T$-invariant when 0 is 2 -adic. Hsia uses characteristic sets and $G$-invariants for vectors and he solves the problem when $o$ is dyadic in general. We define here a new numerical invariant, the degree of a vector, which reflects more on the structure of $L$ and the relationship between $x, y$ and $L$. The Witt conditions will be stated in terms of this degree invariant.

1. Introduction. Let $\pi$ be a prime element generating the maximal ideal of $\mathfrak{D}$ and let $e$ be such that $2 \mathfrak{0}=\pi^{e} \mathfrak{D}$. Let $Q$ be a quadratic form on $L, B$ its associated symmetric bilinear form. Then $Q$ and $B$ are connected by

$$
Q(x+y)=Q(x)+Q(y)-B(x, y) .
$$

The lattice $L$ is unimodular simply means $B(L, L)=\mathfrak{o}$ and $\operatorname{det} L$ is a unit. The structure of unimodular lattices is well-known and can be found in O'Meara [7]. A vector $v$ is primitive if $v \notin \pi L$. Hence $v$ is primitive if and only if $B(v, L)=\mathfrak{o}$.

Proposition 1. Let $v$ be a vector in a unimodular lattice L. Then $v \in \pi^{k} L$ if and only if $B(v, L) \subseteq \pi^{k} \mathrm{D}$.

Proof. The necessity is trivial. Assume $B(v, L) \subseteq \pi^{k} \mathfrak{o}$ and $h$ is the highest power of $\pi$ that divides $v$, that is, $v=\pi^{h} w$ for some primitive vector $w$. Hence $B(w, L)=\mathfrak{0}$ and $B(v, L)=B\left(\pi^{h} w, L\right)=$ $\pi^{h} \mathfrak{D}$ and $h \geqq k$.

If $z \in L$ satisfies ord $Q(z) \leqq$ ord $B(z, L)$, then the map $\sigma_{z}$ given by

$$
\sigma_{z}(v)=v-B(v, z) z / Q(z)
$$

is an integral isometry known as the reflection of $z$. The group of integral isometries is denoted by $O(L)$. O'Meara and Pollak [8], [9] have shown that $O(L)$ is generated by reflections except in a few cases when the residue field $\mathrm{o} / \pi \mathrm{o}$ contains only two elements, and that in the exceptional cases one extra generator given by an 
Eichler transform is needed. If $i$ is a nonzero isotropic vector and $z$ satisfies $B(i, z)=0$ then an Eichler transform $E_{z}^{i}$ is defined by:

$$
E_{z}^{i}(v)=v+B(v, i) z-B(v, z) i-Q(z) B(v, i) i .
$$

We say two vectors $x$ and $y$ are associated, denoted $x \sim y$ if there is a $\phi \in O(L)$ such that $\phi(x)=y$. For each $k$ such that $e \geqq k \geqq 0$, let

$$
L^{(-k)}=\left\{v \in L: Q(v) \in \pi^{-k} \mathfrak{p}\right\} .
$$

Each $L^{(-k)}$ is invariant under the action of $O(L)$ and

$$
L=L^{(-e)} \supseteq \cdots \supseteqq L^{(-1)} \supseteqq L^{(0)} \text {. }
$$

Definition. The lattice $L$ is said to have degree $k$ if

$$
L=\cdots=L^{(-k)} \neq L^{(-k+1)} .
$$

The sublattice $L^{(0)}$ is called the even sublattice of $L$. A degree 0 lattice is simply called an even lattice.

\section{The degree invariant.}

Definition. Let $v$ be a primitive vector. The degree of $v$, $m(v)$, is given by $m(v)=\operatorname{ord} B\left(v, L^{(0)}\right)$.

If $d$ is the degree of $L$, then clearly $\pi^{[d / 2]} L \subseteq L^{(0)}$, where [d/2] denotes the smallest integer greater than $d / 2$. Consequently, $m(v) \leqq$ $[d / 2]$.

Furthermore, if $v$ is a primitive vector with degree $m$ and $w$ is another primitive vector with $w-v \in \pi^{m} L$, then the degree of $w$ is also $m$. For we have $w=v+\pi^{m} z$ and

$$
\begin{aligned}
B\left(w, L^{(0)}\right) & =B\left(v+\pi^{m} z, L^{(0)}\right) \\
& =B\left(v, L^{(0)}\right)+\pi^{m} B\left(z, L^{(0)}\right) \\
& =\pi^{m} \mathcal{D} .
\end{aligned}
$$

\section{Witt's theorem.}

THEOREM. Let $x$ and $y$ be primitive vectors such that $Q(x)=$ $Q(y)$. Then $x$ is associated to $y$ if and only if $m(x)=m(y)=m$ and $y-x \in \pi^{m} L$.

We remark that the condition $y-x \in \pi^{m} L$ expresses how close the vectors $x$ and $y$ must be. With the upperbounds calculated for $m$, this condition becomes quite appealing. Before proving the 
theorem we first set up an invariant which has its own importance.

Definition. If $a$ is an element of $K$, the quotient field of $\mathrm{o}$, let

$$
S_{a}=\{u \in L: Q(u) \equiv a \bmod \mathfrak{o}\} .
$$

Definition. If $v$ is a primitive vector of degree $m$, let

$$
S_{a}(v)=B(v, u) \bmod \pi^{m} \mathfrak{v}
$$

where $u$ is a vector in $S_{a}$.

Since $Q(\phi(u))=Q(u)$ for any isometry $\phi$, it is clear that $S_{a}$ is invariant under $O(L)$. To show that $S_{a}(v)$ is well-defined, let $u, u^{\prime}$ be vectors in $S_{a}$. Then $Q(u) \equiv Q\left(u^{\prime}\right) \equiv a \bmod \mathrm{b}$, and

$$
\begin{aligned}
Q\left(u-u^{\prime}\right) & =Q(u)-Q\left(u^{\prime}\right)-B\left(u^{\prime}, u-u^{\prime}\right) \\
& \equiv 0 \bmod \mathfrak{o} .
\end{aligned}
$$

Hence $u-u^{\prime} \in L^{(0)}$ and

$$
\begin{gathered}
B(v, u)-B\left(v, u^{\prime}\right)=B\left(v, u-u^{\prime}\right) \\
\equiv 0 \bmod \pi^{m} \mathfrak{D} .
\end{gathered}
$$

Since $S_{a}$ is invariant under $O(L)$, we immediately obtain that if $x$ and $y$ are associated, then $S_{a}(x)=S_{a}(y)$.

Proof of theorem. Let $x$ and $y$ be associated vectors. Clearly $m(x)=m(y)$. For each nonempty $S_{a}$, we have

$$
S_{a}(x) \equiv S_{a}(y) \bmod \pi^{m} \mathfrak{D} .
$$

Therefore,

$$
B(y-x, u) \equiv 0 \bmod \pi^{m} \mathfrak{D}
$$

for any $u \in S_{a}$. Since the collection $\left\{S_{a}\right\}$ partitions $L$, this means

$$
B(y-x, u) \equiv 0 \bmod \pi^{m} \mathfrak{D}
$$

for all $u \in L$. Proposition 1 shows that $y-x \in \pi^{m} L$.

It is convenient to collect that following two results.

Proposition 2. Let $x$ and $y$ be primitive vectors such that $Q(x)=Q(y)$ and $m(x)=m(y)=0$. Then $x \sim y$.

Proof. This is a direct application of Kneser's theorem [6]. 
Proposition 3. Let $x$ and $y$ be primitive vectors such that $Q(x)=Q(y)$ and $m(x)=m(y)=m$. Then $x \sim y$ provided one of the following holds:

(i) $y-x \in \pi^{m} L$ and ord $Q(y-x)=2 m$;

(ii) $y-x \in \pi^{m} L$ and there is a vector $u \in L^{(0)}$ with ord $Q(u)=0$ and ord $B(x, u)=$ ord $B(y, u)=m$;

(iii) $y-x \in \pi^{m+1} L$ and there is a vector $u \in L^{(-1)}-L^{(0)}$ with ord $B(x, u)=$ ord $B(y, u)=m$.

Proof. Let $z=\pi^{-m}(y-x)$.

(i ) Since $Q(z)$ is a unit, the reflection $\sigma_{z}$ is integral and sends $x$ to $y$.

(ii) We may assume ord $Q(z)>0$. Let

$$
z^{\prime}=z+B(x, u) u / \pi^{m} Q(u) .
$$

Then it is easily shown that $Q\left(z^{\prime}\right)$ is a unit. Hence $\sigma_{z^{\prime}}$ and $\sigma_{u}$ are integral reflections and $\sigma_{z}, \sigma_{u}(x)=y$.

(iii) Again assume ord $Q(z)>0$. Let

$$
z^{\prime}=z+B(x, u) u / \pi^{m+1} Q(u) .
$$

Then ord $Q\left(z^{\prime}\right)=-1$. Hence $\sigma_{z^{\prime}}$ is integral and $\sigma_{z^{\prime}} \sigma_{u}(x)=y$.

Proof of theorem (continued). Let $x$ and $y$ be primitive vectors satisfying the conditions $Q(x)=Q(y), m(x)=m(y)=m$ and $y-x \epsilon$ $\pi^{m} L$.

If $m=0$, Proposition 2 settles the problem. Let $m \geqq 1$. We may further assume that ord $Q(y-x)>2 m$, otherwise Proposition 3 (ii) already provides the necessary isometry. We proceed with the proof in a series of lemmas.

Lemma 1. If $B\left(x, L^{(-1)}\right)=B\left(y, L^{(-1)}\right)=\pi^{m-1} \mathrm{o}$, then $x \sim y$.

Proof. Since $B\left(x, L^{(0)}\right)=B\left(y, L^{(0)}\right)=\pi^{m}$ o, we know that $L^{(-1)}-$ $L^{(0)}$ is a nonempty set. Choose $v$ and $w$ from this set so that ord $B(x, v)=$ ord $B(y, w)=m-1$. Then one of the vectors $v, w, v+$ $w$, which we denote by $u$, will satisfy ord $B(x, u)=\operatorname{ord} B(y, u)=$ $m-1$. This vector $u$ also lies in $L^{(-1)}-L^{(0)}$. Let $z=\pi^{-m}(y-x)$ and $z^{\prime}=z+B(x, u) u / \pi^{m-1} Q(u)$. Then $\sigma_{z^{\prime}}$ is an integral isometry and $\sigma_{z^{\prime}} \sigma_{u}(x)=y$.

Lemma 2. If $B\left(x, L^{(-2)}\right)=B\left(y, L^{(-2)}\right)=\pi^{m-1} \mathfrak{0}$ and $B\left(x, L^{(-1)}\right)=$ $B\left(y, L^{(-1)}\right)=\pi^{m} \mathfrak{o}$, then $x \sim y$.

Proof. As in Lemma 1, we can choose a vector $z$ from $L^{(-2)}-$ 
$L^{(-1)}$ such that ord $B(x, z)=$ ord $B(y, z)=m-1$. Then Proposition 3 (ii) can be applied with $u=\pi z$.

Lemma 3. Assume $B\left(x, L^{(-2)}\right)=B\left(y, L^{(-2)}\right)=\pi^{m} \mathrm{D}$. If there is a vector $z \in L^{(-2)}-L^{(-1)}$, then $x \sim y$.

Proof. There are vectors $v$ and $w$ in $L^{(0)}$ such that ord $B(x, v)=$ ord $B(y, w)=m$. One of the three vectors $v, w, v+w$, which will be denoted by $u$, must satisfy $\operatorname{ord} B(x, u)=\operatorname{ord} B(y, u)=m$. If ord $Q(u)=0$, Proposition 3 (ii) can be used. Otherwise let $u^{\prime}=u+$ $\pi z$. Then ord $Q\left(u^{\prime}\right)=0$ and $\operatorname{ord} B\left(x, u^{\prime}\right)=\operatorname{ord} B\left(y, u^{\prime}\right)=m$. Hence Proposition 3 (ii) can again be used.

From here on we may assume that $B\left(x, L^{(-2)}\right)=B\left(y, L^{(-2)}\right)=\pi^{m} \mathrm{D}$, and that there are no vectors $u$ in $L$ with ord $Q(u)=-2$. This further means that there are no vectors $u$ in $L$ with $Q(u)$ having negative even orders. Hence the degree of $L$ equals $-2 h+1$ for some positive integer $h$. By an earlier remark and Proposition 2, we may assume that $h>m>1$.

LEMMA 4. Under the above assumptions, the lattice $L$ has one of the following decompositions:

(i ) $L=\mathrm{o} v^{\perp} M$ if $L$ is odd-dimensional,

(ii) $L=(\mathrm{o} v \oplus \mathrm{o} w)^{\perp} M$ if $L$ is even-dimensional, where ord $Q(v)=-2 h+1$, ord $Q(w) \geqq 1, B(v, w)=1$ and $M$ is an even sublattice.

Proof. (i) We can write $L=\mathfrak{o} v_{1} \perp M_{1}$, where ord $Q\left(v_{1}\right)=-2 h+$ 1. If $M_{1}$ is not even, then $M_{1}$ contains vectors $u$ with ord $Q(u)$ being some negative odd integer. By adding appropriate vectors $a v_{1}, a \in \mathcal{D}$, to these vectors, we can form a new decomposition $L=$ $\mathfrak{0} v_{2} \perp M_{2}$ with the degree of $M_{2}$ less than the degree of $M_{1}$. By induction we can obtain the desired decomposition.

(ii) Starting with a decomposition $L=\left(\mathfrak{o} v_{1} \oplus \mathfrak{o} w_{1}\right) \perp M_{1}$, we can use $v_{1}$ to change $M_{1}$ until $L=\left(\mathfrak{o} v_{2} \oplus \mathfrak{o} w_{2}\right) \perp M$, where $M$ is even. Finally, since ord $Q\left(w_{2}\right)$ is odd or greater than 0 , we can use $v_{2}$ to change $w_{2}$ to obtain the desired decomposition.

Lemma 5. Let $L$ be odd-dimensional. Then there exists an isometry $\phi$ such that $\dot{\phi}(x)-y \in \pi^{m+1} L$. Hence $x \sim y$.

Proof. Let $L=\mathrm{o} v \perp M$ be given by Lemma 4 . Write

$$
x=a v+\pi^{m} z, \quad y=b v+\pi^{m} z^{\prime}
$$


where $z, z^{\prime}$ are primitive vectors of $M$. Since

$$
Q(x)-Q(y)=\left(a^{2}-b^{2}\right) Q(v)+\pi^{2 m}\left(Q(z)-Q\left(z^{\prime}\right)\right)=0,
$$

we have

$$
\left(a^{2}-b^{2}\right) Q(v) \equiv 0 \bmod \pi^{2 m} \mathfrak{D}
$$

so that

$$
a^{2}-b^{2} \equiv 0 \bmod \pi^{2 m+2 h-1} \mathfrak{D},
$$

and

$$
a-b \equiv 0 \bmod \pi^{m+h_{\mathfrak{D}}} .
$$

Hence $\pi^{2 m}\left(Q(z)-Q\left(z^{\prime}\right)\right) \equiv 0 \bmod \pi^{2 m+1} \mathfrak{o}$ and

$$
Q(z)-Q\left(z^{\prime}\right) \equiv 0 \bmod \pi \mathrm{0} .
$$

There exists a vector $w \in M$ with $B\left(w, z^{\prime}\right)=1$. Let $u=z^{\prime}+c \pi w$, so that $Q(u)=Q\left(z^{\prime}\right)+c \pi+c^{2} \pi^{2} Q(w)$. The equation

$$
Q\left(z^{\prime}\right)+c \pi+c^{2} \pi^{2} Q(w)=Q(z)
$$

can be solved for $c$ by Hensel's lemma. Since $Q(u)=Q(z), m(u)=$ $m(z)=0$, by Proposition 2 there is an isometry $\phi$ in $O(M)$ such that $\phi(z)=u$. Now $z-u \in \pi^{m+1} L$. By Proposition 3 (iii), $\phi(x) \sim y$ and so $x \sim y$.

LeMMA 6. Let $L$ be even-dimensional. Then $x \sim y$.

Proof. Let $L=(\mathfrak{o} v \oplus \mathfrak{o} w) \perp M$ be given by Lemma 4 . Write

$$
\begin{aligned}
& x=\pi^{m} a v+w+\pi^{m} z \\
& y=\pi^{m} b v+\varepsilon w+\pi^{m} z^{\prime},
\end{aligned}
$$

where $z$ and $z^{\prime}$ are in $M$ and $\varepsilon$ is a unit. Then

$$
\begin{aligned}
0=Q(x)-Q(y)= & \pi^{2 m}\left(a^{2}-b^{2}\right) Q(v)+\pi^{m}(a-b \varepsilon) \\
& +\left(1-\varepsilon^{2}\right) Q(w)+\pi^{2 m}\left(Q(z)-Q\left(z^{\prime}\right)\right) .
\end{aligned}
$$

Using an argument similar to that used in Lemma 5, we show that $a-b \in \pi^{h} \mathfrak{o}$ and $1-\varepsilon \in \pi^{m} \mathfrak{D}$. Hence for some $c \in \mathfrak{D}$,

$$
\begin{aligned}
\pi^{m}(a-b \varepsilon) & =\pi^{m}\left(a-\left(a+\pi^{h} c\right) \varepsilon\right) \\
& \equiv \pi^{m} a(1-\varepsilon) \bmod \pi^{2 m+1} \mathfrak{o} .
\end{aligned}
$$

If $\operatorname{ord}(1-\varepsilon) \geqq m+1$, then $\pi^{m} a(1-\varepsilon) \equiv 0 \bmod \pi^{2 m+1} \mathfrak{D}$. Hence $Q(z)-$ $Q\left(z^{\prime}\right)=0 \bmod \pi \mathrm{D}$ and there is an isometry $\phi \in O(M)$ such that $\phi(z)-$ $z \in \pi M$. Hence $\phi(x)-y \in \pi^{m+1} L$ and $\phi(x) \sim y$ by Proposition 3 (iii). 
If ord $(1-\varepsilon)=m$ we note that $a$ and $b$ must be simultaneously units or nonunits.

(1) Both $a$ and $b$ are units. Then ord $\pi^{m}(a-b \varepsilon)=2 m$. Hence ord $\left(Q(z)-Q\left(z^{\prime}\right)\right)=0$, and at least one of $Q(z), Q\left(z^{\prime}\right)$ is a unit. Without loss of generality, let $Q(z)$ be a unit. If ord $B\left(z, z^{\prime}\right) \geqq 1$, then the vector $u=z+w$ fulfills the hypothesis of Proposition 3 (ii), hence $x \sim y$. Now let ord $B\left(z, z^{\prime}\right)=0$.

(i) ord $Q\left(z^{\prime}\right) \geqq 1$. There exists a vector $z^{\prime \prime}=z+\zeta z^{\prime}$ such that $\zeta$ is a unit and $Q\left(z^{\prime \prime}\right)=Q\left(z^{\prime}\right)$. For this $z^{\prime \prime}$, we have $B\left(z^{\prime \prime}, M\right)=$ $B\left(z^{\prime}, M\right)=$ o. Hence there is an isometry $\phi \in O(M)$ with $\phi\left(z^{\prime}\right)=z^{\prime \prime}$. Proposition 3 (ii) can now be used on $\phi(x)$ and $y$, with $u=z$.

(ii) ord $Q(z)=$ ord $Q\left(z^{\prime}\right)=0$. Since $Q(z)-Q\left(z^{\prime}\right)$ is not zero, the residue field $\mathfrak{v} / \pi \mathfrak{0}$ must possess more than two elements. And since ord $B(x, w+\zeta z)=0$ for all units $\zeta$, we can choose a unit $\zeta$ such that ord $B(y, w+\zeta z)=0$ as well. Now Proposition 3 (ii) can be used with $u=w+\zeta z$.

(2) Both $a$ and $b$ are nonunits. Then ord $a(1-\varepsilon) \geqq 2 m+1$. Here $Q(z)-Q\left(z^{\prime}\right) \equiv 0 \bmod \pi 0$. Hence there is an isometry $\phi \in O(M)$ such that $\phi(z) \equiv z^{\prime} \bmod \pi M$. Now we can rewrite

$$
\begin{aligned}
& x=\pi^{m+1} a^{\prime} v+w+\pi^{m} z \\
& y=\pi^{m+1} b^{\prime} v+\varepsilon w+\pi^{m} z+\pi^{m+1} \bar{z},
\end{aligned}
$$

where $\bar{z} \in M$. Since $x$ and $y$ are primitive, $z$ must also be primitive. Hence there exists a primitive vector $z^{\prime \prime} \in M$ which decomposes $M$ as:

$$
M=\left(\mathfrak{o} z \oplus \mathfrak{o} z^{\prime \prime}\right) \perp M^{\prime} .
$$

If $\operatorname{ord} Q(z) \geqq 1$, we may choose $z^{\prime \prime}$ so that ord $Q\left(z^{\prime \prime}\right)=0$. Hence the hypothesis of Proposition 3 (ii) is satisfied with $u=z^{\prime \prime}+w$. Assume now ord $Q(z)=0$. If we can choose a vector $z^{\prime \prime}$ with ord $Q\left(z^{\prime \prime}\right)=0$, we are again done. Otherwise we can choose a vector $z^{\prime \prime}$ with $Q\left(z^{\prime \prime}\right)=0$. Let $\gamma=(\varepsilon-1) / \pi^{m}$. Consider the Eichler transform $E_{\gamma w}^{z^{\prime \prime}}$ on $x$ :

$$
E_{\gamma w}^{z^{\prime \prime}(x)}=x+B\left(x, z^{\prime \prime}\right) \gamma w-B(x, \gamma w) z^{\prime \prime}-Q(\gamma w) B\left(x, z^{\prime \prime}\right) z^{\prime \prime} .
$$

An easy calculation shows that

$$
x-E_{\gamma w}^{z^{\prime \prime}}(x) \equiv \varepsilon w \bmod \pi^{m+1} L .
$$

Hence

$$
y-E_{\gamma w}^{z^{\prime \prime}}(x) \equiv 0 \bmod \pi^{m+1} L .
$$

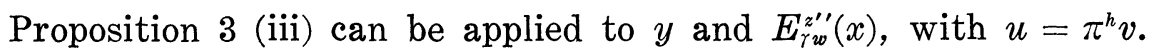




\section{REFERENCES}

1. J. S. Hsia, An invariant for integral equivalence, Amer. J. Math., 93 (1971), 867-871.

2. Integral equivalence of vectors over depleted modular lattices on dyadic local fields, Amer. J. Math., 90 (1968), 285-294.

3 . - Integral equivalence of vectors over local modular lattices, Pacific J. Math., 23 (1967), 527-542.

4. - Integral equivalence of vectors over local modular lattice, II, Pacific J. Math., 31 (1969), 47-59.

5. D. G. James, On Witt's theorem for unimodular quadratic forms, II, Pacific J. Math., 33 (1970), 645-652.

6. M. Kneser, Witt's Satz für quadratische Formen über lokalen Ringen, Nach. der Akad. Wiss. Göttingen, II, Math-Phys. Klasse, Heft, 9 (1972), 195-205.

7. O. T. O'Meara, Introduction to Quadratic Forms, Springer Verlag, 1962.

8. O. T. O'Meara and B. Pollak, Generation of local integral orthogonal groups, Math. Zeit., 87 (1965), 385-400.

9. - Generation of local integral orthogonal groups, II, Math. Zeit., 93 (1966), 171-188.

10. A. Trojan, The integral extension of isometries of quadratic forms over local fields, Canad. J. Math., 18 (1966), 920-942.

Received August 31, 1977.

MASSAChuSETTS INSTITUTE OF TEChNOLOGY

CAMBRIDGE, MA 02139 


\section{PACIFIC JOURNAL OF MATHEMATICS}

\section{EDITORS}

RICHARD ARENS (Managing Editor)

University of California

Los Angeles, CA 90024

Charles W. Curtis

University of Oregon

Eugene, OR 97403

C. C. MOORE

University of California

Berkeley, CA 94720
J. DUGUNDJI

Department of Mathematics

University of Southern California

Los Angeles, CA 90007

R. FINN and J. MILGRAM

Stanford University

Stanford, CA 94305

\section{ASSOCIATE EDITORS}

\section{E. F. BeCKENBACH}

B. H. NeumanN

F. WOLF

K. YoSHIDA

\section{SUPPORTING INSTITUTIONS}

\author{
UNIVERSITY OF SOUTHERN CALIFORNIA \\ STANFORD UNIVERSITY \\ UNIVERSITY OF HAWAII \\ UNIVERSITY OF TOKYO \\ UNIVERSITY OF UTAH \\ WASHINGTON STATE UNIVERSITY \\ UNIVERSITY OF WASHINGTON
}

The Supporting Institutions listed above contribute to the cost of publication of this Journal, but they are not owners or publishers and have no responsibility for its content or policies.

Mathematical papers intended for publication in the Pacific Journal of Mathematics should be in typed form or offset-reproduced, (not dittoed), double spaced with large margins. Please do not use built up fractions in the text of the manuscript. However, you may use them in the displayed equations. Underline Greek letters in red, German in green, and script in blue. The first paragraph or two must be capable of being used separately as a synopsis of the entire paper. Items of the bibliography should not be cited there unless absolutely necessary, in which case they must be identified by author and journal, rather than by item number. Manuscripts, in triplicate, may be sent to any one of the editors. Please classify according to the scheme of Math. Reviews, Index to Vol. 39. All other communications should be addressed to the managing editor, or Elaine Barth, University of California, Los Angeles, California, 90024.

50 reprints to each author are provided free for each article, only if page charges have been substantially paid. Additional copies may be obtained at cost in multiples of 50 .

The Pacific Journal of Mathematics is issued monthly as of January 1966. Regular subscription rate: $\$ 72.00$ a year (6 Vols., 12 issues). Special rate: $\$ 36.00$ a year to individual members of supporting institutions.

Subscriptions, orders for numbers issued in the last three calendar years, and changes of address should be sent to Pacific Journal of Mathematics, P.O. Box 969, Carmel Valley, CA 93924, U.S.A. Older back numbers obtainable from Kraus Periodicals Co., Route 100, Millwood, NY 10546.

PUBLISHED BY PACIFIC JOURNAL OF MATHEMATICS, A NON-PROFIT CORPORATION

Printed at Kokusai Bunken Insatsusha (International Academic Printing Co., Ltd.). 8-8, 3-chome, Takadanobaba, Shinjuku-ku, Tokyo 160, Japan. 


\section{Pacific Journal of Mathematics}

\section{Vol. 80, No. $2 \quad$ October, 1979}

K. Adachi, On the multiplicative Cousin problems for $N^{p}(D) \ldots \ldots \ldots \ldots 297$

Howard Banilower, Isomorphisms and simultaneous extensions in $C(S) \ldots 305$

B. R. Bhonsle and R. A. Prabhu, An inversion formula for a distributional

finite-Hankel-Laplace transformation ................... 313

Douglas S. Bridges, Connectivity properties of metric spaces.......... 325

John Patton Burgess, A selection theorem for group actions ........... 333

Carl Claudius Cowen, Commutants and the operator equations

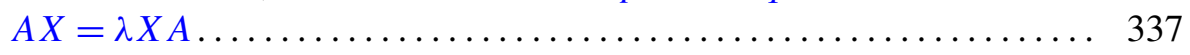

Thomas Curtis Craven, Characterizing reduced Witt rings. II .......... 341

J. Csima, Embedding partial idempotent d-ary quasigroups ............ 351

Sheldon Davis, A cushioning-type weak covering property ............ 359

Micheal Neal Dyer, Nonminimal roots in homotopy trees ............. 371

John Erik Fornaess, Plurisubharmonic defining functions ........... 381

John Fuelberth and James J. Kuzmanovich, On the structure of finitely

generated splitting rings .......................... 389

Irving Leonard Glicksberg, Boundary continuity of some holomorphic

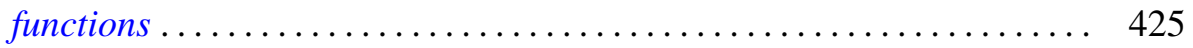

Frank Harary and Robert William Robinson, Generalized Ramsey theory.

IX. Isomorphic factorizations. IV. Isomorphic Ramsey numbers .......

Frank Harary and Allen John Carl Schwenk, The spectral approach to determining the number of walks in a graph...........

David Kent Harrison, Double coset and orbit spaces ..... . .

Shiro Ishikawa, Common fixed points and iteration of commuting

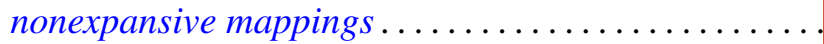

Philip G. Laird, On characterizations of exponential polynomials ........ 503

Y. C. Lee, A Witt's theorem for unimodular lattices ...........

Teck Cheong Lim, On common fixed point sets of commutative

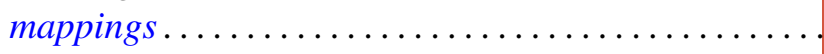

R. S. Pathak, On the Meijer transform of generalized functions ...

T. S. Ravisankar and U. S. Shukla, Structure of $\Gamma$-rings . . .

Olaf von Grudzinski, Examples of solvable and nonsolvable convolution

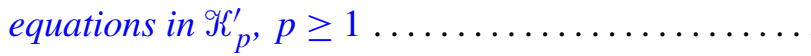

